\title{
»Die wirkliche Welt auf wirklichen Bildschirmen«
}

\author{
Fernsehen in Andreas Neumeisters \\ Angela Davis löscht ihre Website
}

ANNA-CARINA MEYWIRTH

2002 veröffentlicht der >Suhrkamp-Autor $<$ Andreas Neumeister Angela Davis löscht ihre Website. Nicht beispielsweise als Roman, sondern als »Listen, Abbildungen [und] Refrains « wird das Buch im Untertitel angekündigt. ${ }^{1}$ Mit Fragmenten aus u.a. Fernsehshows, Nachrichtensendungen, Liedtexten und Werbeslogans spielt der Autor auf 121 Seiten und gibt einen Rückblick in die Programmgestaltung um das Jahr 2000 (Big Brother, Zlatkos Welt, Veronas Welt und Peep!). Das gesammelte Fernsehmaterial, popästhetisch verarbeitet und kommentiert, offenbart Neumeisters politische Auseinandersetzung mit scheinbaren (Fernseh-)Banalitäten und kippt die ebenso scheinbar klar definierte Trennung von »fiction« und »non-fiction« (AD: 45). Auf welche höchst implizite Weise dies in Angela Davis geschieht, wird im Folgenden dargelegt.

\section{I »Täuschend falsch wirkt einleuchtend echt ist aber falsch ${ }^{2}$}

»Kameras filmen Kameras, wie sie Kameras filmen

Schlafzimmer der Frauen

Dusche

Tagebuchzimmer

1 Neumeisters Angela Davis löscht ihre Website (2002) wird im Folgenden mit AD abgekürzt.

2 AD: 37. 
Versorgungsschleuse

Schlafzimmer der Männer

Hühnerstall« (AD: 20)

Neumeister nimmt mit diesem Zitat Rekurs auf die zwischen den Jahren 2000 und 2011 in Deutschland ausgestrahlte Reality-Show Big Brother, die aufgrund ihres großen Publikumserfolgs in zahlreichen Ländern der Welt produziert wurde. War die ursprünglich niederländische Sendung in den ersten Produktionsjahren quasi omnipräsent in den Medien, so schien sie zuletzt umso rapider in Vergessenheit geraten zu sein. Die Kritik gegen die durch das Sendeformat vorangetriebene Enttabuisierung ${ }^{3}$ des Fernsehens (vorwiegend aufgrund der uneingeschränkten Kameraüberwachung der Containerbewohner) war zwar laut, die Einschaltquoten aber übertrafen diese Empörung um ein Vielfaches: Big Brother, welches jeweils 100 Tage das alltägliche Leben der Container-Bewohner zeigte, war ein Kassenschlager, der in vielerlei Hinsicht bisherige Grenzen überschritt. Die Bezeichnung der Rezipienten als »Schaulustige « (AD: 20) könnte in Angela Davis insofern nicht besser gewählt sein. Spätestens mit dieser voyeuristischen Produktion kann Postmans Zuschreibung, dass das Fernsehen ein Medium der totalen Enthüllung sei (vgl. Postman 1996), - so scheint es zunächst nicht mehr negiert werden. Die Show löste nicht nur die Trennung von Privatheit und Öffentlichkeit auf (vgl. Schwering/Stäheli 2000: 11f.), sondern simulierte zudem, die Unterscheidung von Realität und medial inszenierter Wirklichkeit aufzuheben (vgl. Stäheli 2000: 67-70). Versuche, das Sendeformat aufgrund menschenunwürdiger Zustände abzusetzen, scheiterten an der Popularität und dem immensen Medienecho (vgl. Goldbeck/Kassel 2000: 234f.). Dass Kritik an der Sendung und Fragen zu dem Konzept erfolgreich mit Gegenfragen abgewehrt wurden, wird von Neumeister als zweifelhaftes Vorgehen des Senders mehrfach kritisiert:

»Gegenfrage: zwingen wir die Kandidaten etwa

an der Show teilzunehmen?

we know, how to entertain you

six billion humans can't be wrong«(AD: 27)

Neumeisters Collage, welche diese vor nunmehr 15 Jahren geführte, hochbrisante Diskussion verarbeitet, ist keine simple Zitat-Kompilation, vielmehr tritt mit-

3 Dieser Begriff wurde laut Duden erst aufgrund der Diskussion um Big Brother populär (vgl. Wills 2001: 177). 
tels Permutation und minimalistisch formulierter Gedankenfetzen eine Medienkritik zutage, die insbesondere Machtverhältnisse, Boulevardisierungen und Realitätskonstruktionen des Fernsehens in den Fokus rückt. Für letztere wird Big Brother zum Exempel: Welche $>$ Reality< wird dem Zuschauer angeboten? »[D]a real world « (AD: 15) oder nur eine »vermeintlich tatsächlich[e]« (AD: 17)? Ist es »fiction, non-fiction, non-fictive fiction?«(AD: 20) Das unauffällige und gleichzeitig refrainartig wiederkehrende »you decide« zwischen den Textblöcken ermuntert dazu, die medial dargebotene Wirklichkeit zu reflektieren und Position zu beziehen. An die Stelle von TV-Berieselung tritt im Text eine aktive Rezeption.

Abbildung 1: Kameralinse mit Big Brother-Logo

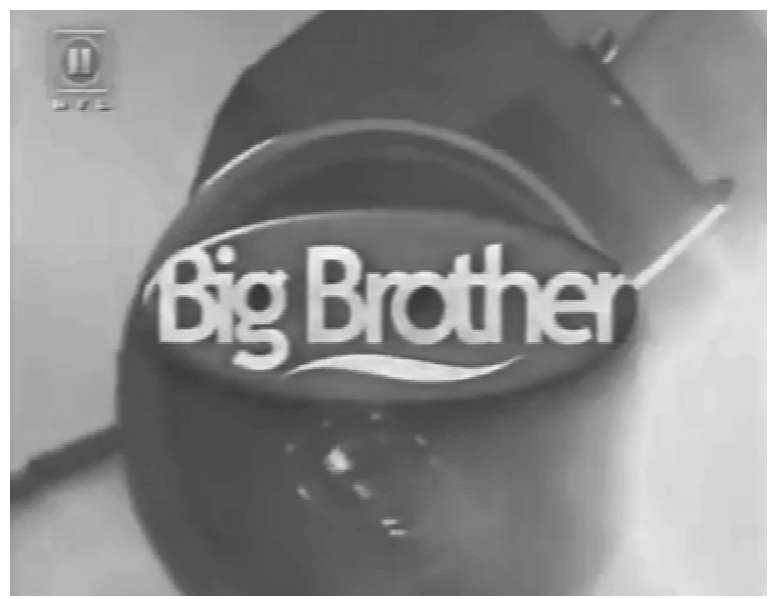

Quelle: YouTube

Selbst wenn der Sender vorgab, ausschließlich das >wahre $<$ Big-Brother-Leben der Containerbewohner zu zeigen und dies mit den 24-stündigen Liveübertragungen $\mathrm{zu}>$ beweisen $<$ versuchte, ist diese Aussage selbstredend absurd. Denn schon mit der Auswahl der Kameraperspektive und dem zusammengeschnittenen Tagesrückblick hat der Rezipient es mit einer mittelbaren Darstellung zu tun, die Meinungsbildungen lenken und Inszenierungsarbeit leisten kann (vgl. Hickethier/Bleicher 1998: 371f.). Suggerieren Werbeslogans oder gar Genrebezeichnungen im Fernsehen die Reproduktion von >wirklicher Realität<, so war diese in der Fernsehgeschichte nie das primäre Ziel. Von Beginn an, folglich ab den 1950er Jahren in Deutschland, standen die Popularisierung bedeutender Ereignisse, der Gewinn eines möglichst breiten Publikums und damit einhergehend möglichst hohe Einschaltquoten im Vordergrund. So trugen 1953 die Krönung 
von Königin Elisabeth II. und 1954 die Fußballweltmeisterschaft in hohem Maße dazu bei, diese Vorhaben innerhalb kurzer Zeit weitgehend zu realisieren (vgl. Böhn/Seidler 2014: 129). Mit der Inszenierung alltäglicher Sujets, welche ein weitaus höheres Identifikationspotenzial aufweisen als singuläre Massenereignisse, ließ sich die Film- und Fernsehlust der Bevölkerung weiter stimulieren. Ab den 1960er Jahren konnte sich nicht einmal mehr der >Underground < mit seinen alternativen Themen und Filmproduktionen von den Projekten der Filmindustrie abgrenzen, da dieser gewahr wurde, dass sich mit subkulturellen Themen der Profit steigern ließ. Gerade der Underground gehörte zuvor, wie Andy Warhol in POPism berichtet, »zu den ganz wenigen Orten, wo man etwas über verbotene Sujets und realistische Szenen des modernen Lebens sehen konnte. Doch jetzt, wo sich Hollywood [...] mit den denselben Themen befasste, wurde die Sache unübersichtlich.« (Warhol/Hackett 2013: 154) Alle wesentlichen Merkmale des Undergroundfilms - neue, freie, >reale $<$ Inhalte - wurden in der Folge von großen Kinoproduktionen adaptiert und nahmen »dem Underground die Attraktivität« aufgrund der »optisch bessere[n] Version« (ebd.). Um mit dieser Qualität konkurrieren zu können, bedurfte es einer modernen technischen Ausstattung, die beispielsweise Warhols Factory nicht zur Verfügung stand. Das Publikum aber, versichert Warhol, würde diese optisch bessere Version der Filmindustrie in jedem Fall bevorzugen. Denn: »Sie war weniger bedrohlich.« (Ebd.) Auf Kosten der >schockierenden Realität< wird ein Film dementsprechend solange ästhetisch aufbereitet, bis er massentauglich ist und damit einhergehend eine entfremdete, weniger >bedrohliche< Realität präsentiert. Dass subversives Potenzial dadurch gänzlich verloren geht und dass Warhol sich womöglich gerade aus diesem Grund für die Nutzung älterer Technik - und daher für einen bewussten ästhetischen Bruch - entschied, sei nur der Vollständigkeit halber erwähnt. »Wir hätten ihnen den Film ganz real gemacht«, kommentiert Warhol abschließend, »[d]amals begriff ich noch nicht, dass sie mit Realität die Kinorealität meinten!« (Ebd.: 155)

Popularität und Alltäglichkeit, so ist in diesem Fall zu schlussfolgern, bedingen sich gegenseitig. Also >reality sells $<$ ? Zumindest lässt sich diese These durch den Boom des Reality-TV (Reality Soaps, Reality Shows) in den 1990ern stützen und auch im letzten Jahrzehnt entstanden weitere Reality-Formate wie die Scripted Reality, deren Name bereits darauf hinweist, dass es sich um eine konstruierte, eine Realität >nach Skript $<$ handelt. ${ }^{4}$ Besonders betont wird dadurch die Tatsache, dass der Terminus $>$ Reality-TV $<$ ein widersinniger ist, denn Wirklichkeit und Fernsehen zusammen zu denken erscheint schon von vornherein para-

4 Vgl. zur Scripted Reality auch Achim Barschs Artikel in diesem Band. 
dox (vgl. Engell 2012: 63ff.). Neumeister weist in Angela Davis auf eben diese Kontradiktion hin und rollt den medialen Wirklichkeitsbegriff, der in eine Medienkritik mündet, neu auf. Die Andeutung von Bildmaterial, das letztlich aber gar nicht vorhanden ist, nimmt dabei eine zentrale Funktion ein. Durch Bilder werde nämlich die Realitätskonstruktion und die emotionale Einbindung der Zuschauer verstärkt. Nicht mehr die Information steht somit im Vordergrund, vielmehr wird diese permanent mit Unterhaltungselementen gekoppelt, um den Rezipienten zufriedenzustellen: »I'm informed. I'm entertained. I'm infotained. I'm okay.« (AD: 40) Gleich auf zwei Buchseiten flimmert diese Aussage dem Leser entgegen und erinnert dabei an das Intro der populären Zeichentricksrie Die Simpsons, in welcher Bart Simpson als Strafarbeit die Tafel mit dem immer gleichen Merksatz beschreibt.

»I'm informed, I'm entertained, I'm infotained, I'm okay

I'm informed, I'm entertained, I'm infotained, I'm okay

I'm informed, I'm entertained, I'm infotained, I'm okay

I'm informed, I'm entertained, I'm infotained, I'm okay

I'm informed, I'm entertained, I'm infotained, I'm okay

I'm informed, I'm entertained, I'm infotained, I'm okay

I'm informed, I'm entertained, I'm infotained, I'm okay

I'm informed, I'm entertained, I'm infotained, I'm okay« (AD: 40)

Visuelle Auffälligkeiten wie diese illustrieren das aufwändige Arrangement des Textes, in welchem sich zentrale popästhetische Verfahren ausmachen lassen: Copy and Paste, Sampling, Collage/Montage, Serialisierung, Cut up. Dazwischen: Leerzeilen, die zugleich als Leerstellen mitgedacht werden können und es Neumeisters präzise konzipiertem Erzähler erlauben über längere Passagen ohne vollständige Sätze oder Überleitungen auszukommen. Infolgedessen stellt sich eine Art Textrhythmus ein, der sowohl klanglich durch wiederholte oder leicht veränderte Phrasen entsteht als auch optisch durch die eben erwähnte, spezielle Anordnung der Textbruchstücke. Was daraus resultiert, ist ein deutungsoffener, »produzierbarer Text« mit »losen Enden«, dessen Lücken im wahrsten Sinne des Wortes »groß genug [sind], um ganze neue Texte in diesen entstehen zu lassen« (Fiske 2008: 42). Inhaltliche Zusammenhänge ergeben sich folglich nicht ausschließlich durch das, was gesagt wird, sondern ebenso durch die intertextuellen Verweise aus dem Text hinaus. Die krasse sprachliche Reduktion ist neben der Absenz von Handlung und Figuren sicherlich ein Grund dafür, warum sich der Erzähler so mühsam konturieren lässt. Zu erkennen gibt er sich zwischen den Zitaten nämlich primär durch seine Fragen, die ebenso banal (»do you like 
horses? « [AD: 69]) wie philosophisch (»gibt es die neue Weltordnung schon?« [AD: 16]) sein können. Ob die regelmäßig auftauchenden Fragen, durch welche der Leser sich automatisch angesprochen fühlt, ganz gleich ob er der intendierte Adressat ist oder nicht, das Ergebnis von verarbeiteten Medienzitaten oder aber tatsächlich ernst gemeinten Äußerungen der textinternen Stimme selbst sind, kann nicht abschließend beantwortet werden. Dessen ungeachtet stechen die Fragezeichen optisch wie Warnsignale aus dem übrigen Text heraus - wie es scheint, um den Leser von Zeit zu Zeit wachzurütteln und ihn mit jener Uneindeutigkeit zu konfrontieren.

»where do you want to go today?«(AD: 15)

»gibt es Jugoslawien völkerrechtlich überhaupt noch? (AD: 16)

»how did you enjoy the war?«(AD: 19)

»what are you waiting for?«(AD: 119)

Es ist offensichtlich, dass der Erzähler sich somit keinesfalls als moralische Autorität präsentiert, wohl aber als Sortierender, Aussuchender, Kommentierender eine nicht unwichtige Position im Text einnimmt, denn durch die kurzen und gleichermaßen pointierten Unterbrechungen der Zitatblöcke zeichnet sich eine vage Positionierung des Ich zur massenmedialen Wirklichkeit ab.

»die Vortäuschung falscher Tatsachen kann eindeutig kein

Straftatbestand sein, allenfalls das Vortäuschen richtiger

Tatsachen, was eben dem Vortäuschen von Tatsachen entspricht, kann unter Umständen ein Straftatbestand sein

you decide«(AD: 22)

So diagnostiziert der Erzähler folgerichtig:

»die Bilder müssen scharf sein, die Wahrheit nur plausibel: stets kreisen fremde Weltbilder getarnt als die eigenen Weltbilder um eine unsichtbare Achse, Zeitmaschinen, Geschichtskompressoren, ein wesentlicher Unterschied zu 1960 ist die massenhafte Verfügbarkeit an Informationen, die in ihrer Fülle jede Zensur überflüssig machen // die eigene Bildwelt formt sich in einer spiralförmigen Drehbewegung aus allen verfügbaren Weltbildern zum eigenen Weltbild $[\ldots] \ll(\mathrm{AD}: 18)$ 
An dieser Stelle wird eine Hierarchisierung von Bild und Wahrheit offengelegt, die ebenso plausibel wie frappierend ist. Nicht die Reliabilität des Gesagten ist von primärer Bedeutung, sondern einzig die Qualität der visuellen Inszenierung. Bilder können somit das Gesagte um weitere Deutungsebenen ergänzen, was Chance und Risiko zugleich bedeutet: »[I]mmer hat es mehrere Kommunikationsebenen gegeben / [...] immer hat es mehr als eine Verständnisebene gegeben.«(AD: 22) Das eigene »Weltbild« entsteht dem Text folgend als Resultat einer massenhaft rezipierten, strudelartigen Bilderflut. Im Sinne Umberto Ecos und Jean Baudrillards hat der Zuschauer es mit einer »Hyperrealität« zu tun, der Simulation einer in dieser Form nicht existierenden Wirklichkeit. Ereignisse werden mithilfe filmischer Mittel verkettet, um Sinn und Kohärenz zu stiften (Baudrillard 1994: 30). Die Nachahmung der Wirklichkeit ist dabei so exakt, dass die Unterscheidbarkeit von virtueller Realität und >wirklicher < Realität verschwimmt und der Zuschauer dem (Irr-)Glauben an eine tatsächliche Realität aufsitzt. Bestärkt wird diese Annahme dadurch, dass nicht wie zu Beginn der Fernsehgeschichte über unabhängige Ereignisse im TV berichtet wird, hingegen aber Events gezielt darauf ausgerichtet sind, um im Fernsehen übertragen zu werden (vgl. Hickethier/Bleicher 1998: 381f.). Die Unterscheidungen von Signifikat und Signifikant oder Kopie und Original werden damit obsolet, denn sobald Bild und Realität »[i]neinanderstürzen«, verschmelzen sie »in einem gemeinsamen Bereich der Simulation « (Engell 2012: 79). Neumeisters Text illustriert dies deutlich, indem das Gesehene permanent angezweifelt wird. Dieses unablässige >Infragestellen< kann infolgedessen als Programmatik des Textes gelesen werden.

»wenn alles wirklich ist, dann besteht die wirkliche Welt aus wirklichen Bildpunkten auf wirklichen Bildschirmen in wirklichen Räumen

Vortäuschung falscher Tatsachen oder Vortäuschung richtiger Tatsachen?

believe the hype! (AD: 22)

Der Textausschnitt erinnert an eine Gleichung, aus welcher sich der Begriff Wirklichkeit >wegkürzen < lässt. Übrig bleibt schließlich die »Vortäuschung«, die Inszenierung, die Show. In Angela Davis findet daher eine literarische Übersetzung der Baudrillardschen Simulationsthese statt: »alles echt / alles relativ / alles relativ falsch // alles falsch / alles relativ / alles relativ echt« (AD: 22). Es 
ist ein »System, in dem wir leben«, ein »Sendegebiet, in dem wir leben « (AD: 14). Der durch Montage erreichten Kohärenz und Logik der BildschirmWirklichkeit kann dabei nicht mehr vertraut werden, vielmehr tritt an diese Stelle des Vertrauens ein Realitätsglaube, mit welchem sich die unaufhörliche Frage, ob nun >falsche oder richtige Tatsachen< vorgetäuscht wurden, erübrigt. Subjektive Realität, so lautet die naheliegende Schlussfolgerung, ist eng mit dem Massenmedium Fernsehen verknüpft und abhängig von demselben.

\section{II »More information? Click here! ${ }^{5}$}

Die Diskussion um die antithetischen Begriffe > wirkliche Realität< und > virtuelle Realität< ist alles andere als ein Novum, aber dennoch ein Feld, das an Aktualität und Diskussionspotenzial nicht eingebüßt hat. 2008 veröffentlicht Rainald Goetz in Klage seine harsche Kritik an TV-Inhalten und indiziert, dass jede Vorenthaltung von Informationen für den Rezipienten eine Verfälschung der Wirklichkeit bedeute und eine Form von Macht demonstriere (vgl. Greif 2004: 269f.). Die komplexe Realität würde dabei zugunsten der Massen auf ein Minimum reduziert (vgl. Fiske 2005) und permanent repetiert, sodass jegliches Irritationspotenzial verloren gehe und das »hochagile Sehen « in eine »lethargische Kurzsichtigkeit« und Apathie umschlage (Greif 2004: 259). Goetz' Gegenvorschlag lautet: Uneindeutigkeit! Denn genau diese setze den Rezipienten frei und sei gleichsam das Synonym für das »Interesse an Wahrheit« (Goetz 2008: 86 u. 75). Allein mit dieser Wahrheit ließen sich ästhetische Ergebnisse hervorbringen, ohne dass eine Beschönigung notwendig wäre, wie sie vom Fernsehen permanent vorgenommen wird (vgl. ebd.: 86). Dieses offenbare Desinteresse der Medien an Authentizität zeigt, dass reale Ereignisse nicht im Fokus stehen, sondern lediglich eine weniger schockierende, flachere Variation derselben; »für die Leute geht es vor allem darum, sich in angenehmer Atmosphäre zu entspannen « (AD: 25). Diese daraus resultierende Simplizität medialer Darstellungen beanstanden die Linguisten Edward Sapir und Benjamin Whorf (vgl. Kloock/Spahr 2012: 114), indem sie reklamieren, dass die visuelle Aufbereitung und Vereinfachung von (insbesondere politischen) Texten eine gezielte Beeinflussung der Meinungsbildung zur Folge haben könne. Sie sprechen sich somit für eine Schriftkultur aus, welche die Wahrnehmung konzentriere und nicht durch Bilder ablenke, um zu emotionalisieren und zu banalisieren. Politik - und demzufolge auch

5 AD: 56. 
andere medial inszenierte Texte - seien »unter dem Leitmedium Fernsehen [...] nicht mehr logisch, sondern psychologisch« (ebd.: 115).

Während Goetz diese extreme Positionierung teilt, indem er ebenfalls der Schrift mehr Reliabilität zuschreibt als manipulierten Text-Bild-Kombinationen >irgendwelcher Fernsehleute< und somit der Banalitätskultur eine Absage erteilt, kommt Neumeisters Text auf den ersten Blick vergleichsweise pädagogisch daher. Jeder Lesende oder Fernsehende ist auf das eigene Kontrollieren angewiesen, da keine konkrete Regieanweisung aus dem Off existiert, die Informationen filtert, zensiert oder bebildert. Obwohl in Angela Davis einerseits allein durch die Textauswahl eine Art >Vorzensur $<$ des Erzählers stattfindet, die Positionen oder Lösungen andeutet, wartet man andererseits vergebens auf ein endgültiges Urteil. Dieses >Sich-selbst-überlassen-Werden $<$ zeugt einmal mehr von dem »emanzipatorisch-antitotalitären Kern« (Rüdenauer 2001), den Pop hat. Obwohl Neumeister in Angela Davis löscht ihre Website weniger in die Offensive geht als Goetz, stehen beide sich in nichts nach und verschreiben sich der Uneindeutigkeit. Anstelle reiner Zitate aus den von Goetz verunglimpften Sendungen, deren Inhalte bis zur Substanzlosigkeit für ein Massenpublikum >aufbereitet< werden, findet der Leser bei Angela Davis weder kausal-logische Verknüpfungen noch weitestgehend eindeutige Lesarten. Infolgedessen kann er - im Gegensatz zum Fernsehzuschauer - nicht alle Informationen sortiert und bebildert auf sich wirken und sich sozusagen >entertainen< lassen. Vielmehr wird der Rezipient durch die Collagenhaftigkeit von Neumeisters Text »entautomatisiert « (Weingart 2005: 246) und in seiner Passivität gestört. Textbruchstücke müssen geordnet werden, was die >Produzierbarkeit< des Textes demonstriert und den Leser als »zentralen Bezugspunkt« (Mikos 2001: 365) definiert. Dem Rezipienten bleibt also die Entscheidung überlassen, ob er sich der Simulation hingibt oder ob er das Spiel entlarvt und wie der Erzähler zu dem Ergebnis kommt: »täuschend falsch wirkt einleuchtend echt ist aber falsch« (AD: 37).

Die Erzählinstanz in Angela Davis scheint dem Leser die Kontrolle zu entziehen und das Hin- und Herschalten zwischen Textelementen zu übernehmen. Der dadurch entstehende Effekt des abrupten Themenwechsels ist gleichzusetzen mit dem des Zappens, des Umschaltens von einem Programm zum nächsten:

»Bohlen rettet Berliner Polizeipferde vor Todesspritze

Bohlen sagt: sind super Tiere

stimmen Sie ab: dürfen Politiker ihre Haare färben?

schreiben Sie ihre Meinung ans Board!«(AD: 98) 
Nicht nur dieses Zapping, auch der zu Beginn auftauchende Text »ein/aus« (AD: 10) auf einer ansonsten leeren Seite in Angela Davis vermittelt dem Rezipienten eher das Gefühl des Fernsehens als das des Lesens. Er sieht sozusagen fern, indem er liest oder um es mit den Worten Fiskes zu formulieren: He is »reading television «. ${ }^{6}$ Im Gegensatz zum tatsächlichen Fernsehen existieren bei Neumeister allerdings einige zentrale Unterschiede: Erstens findet eine bewusst skurrile Kompilation der Fernsehtexte statt, die nicht >weggeschaltet<, also ausgeblendet, werden können. Die zuvor erläuterte, konstruierte Kohärenz des Fernsehprogramms wird somit wieder aufgehoben. Zweitens verschwindet der ephemere Charakter des Fernsehens durch die visuelle Abbildung des Fernsehtextes, denn der Rezipient hat die Möglichkeit vor- und zurückzublättern. Drittens hat >Neumeisters Fernsehen< keine bardische Funktion ${ }^{7}$, wie sie in Reading television beschrieben wird: Zwar reduziert Neumeister die Quantität des Gesagten, jedoch verlieren die Inhalte dadurch nicht an Komplexität. Dies führt automatisch dazu, dass die >Bedürfnisse< der Rezipienten, nämlich »emotional wirksame kulturelle Mythen und Stereotypen « (Renger 2009: 229), keine Priorität darstellen und nicht bedient werden, so wie es laut Fiske eigentlich sein müsste (vgl. Fiske 2005: 85-100). Viertens eliminiert Neumeister den visuellen Kanal vollständig. Während sich alle Medien zu einer immer höheren Bildkonzentration bewegen, verweigert sich Neumeister ihr. Anstatt visuelles Material in seinen Text zu integrieren, beschränkt er sich auf dessen Beschreibung bzw. auf die schlichte Nennung der Untertitel, die jeweils durch »Abb.« gekennzeichnet werden. Jene Bilder, auf die mit dem Kürzel referiert wird, sind dabei tatsächlich teilweise im Internet zu finden. Mit diesen Abbildungskürzeln evoziert Neumeister geradezu ein Bedürfnis, das zugehörige Bild zu sehen, beispielsweise eine TomahawkRakete, die »direkt aus dem All in den Lichtschacht eines Luftfahrtministeriums« (AD: 45) stürzt. Dieses Verlangen nach spektakulären Bildern bewirkt, dass »gerade vom Allerhäßlichsten [...] nur die allerschönsten Bilder gezeigt werden« (ebd.) dürfen und verpönt somit die »[s]chaulustigen[n] (AD: 43) Zuschauer, deren Sensationslust stets vom Medium Fernsehen gestillt wird. Die Absenz der Abbildungen in Angela Davis hingegen deutet an, dass es eines emanzipierten Lesers bedarf: Ohne navigiert, wenn nicht gar manipuliert zu

6 In Anlehnung an den Titel von John Fiske (2005).

7 Wie der Barde in früheren Jahrhunderten hat das Fernsehen laut Fiske und Hartley heute die Funktion Informationen auszuwählen, zu reduzieren und gemäß den jeweiligen kulturellen Bedürfnissen an die audience zu übermitteln. Dies geschieht jeweils mündlich. Da sich sowohl der Barde als auch das Fernsehen an den Kodes der audience orientieren, wird diese zur Autorität (vgl. Fiske 2005: 65). 
werden durch bewusst herbeigeführte Emotionen muss er sich autonom im Textgeflecht orientieren.

Wenn laut Weingart (Fernseh-)Bilder als »Garant für Massentauglichkeit « (Weingart 2005: 218) gelten und damit zu den zentralen Strategien des (Boulevard-)Fernsehens gehören, um eine möglichst hohe Zuschauerbindung zu erreichen (vgl. Brichta 2010: 213), dann ist daraus abzuleiten, dass >Neumeisters bilderloses Fernsehen< nicht für Massen zugeschnitten ist, wohl aber seine Inhalte daraus bezieht und sie transformiert. Sein Text ist insofern das »kritische[] Korrektiv« des Mediums Fernsehen (Schuhmacher 2003: 163). Ein von Eckhart Schumacher formuliertes Resümee beschreibt Andreas Neumeisters Poetik sehr treffend:

»[E]s werden auch Schreibverfahren entwickelt, die sich auf andere Weise dem annähern, was > für alle < zugänglich ist. Gesten hedonistischer Affirmation finden sich in diesem $\mathrm{Zu}$ sammenhang ebenso wie subversive Umfunktionierungen und ideologiekritische Entlarvungen, aber fast nie wird >das Populäre < über Schlagworte wie Simplifizierung oder Verflachung einfach abgetan. [...] Trivialmythen werden [...] in neue Zusammenhänge überführt [...], so dass sich [...] ein Irritationspotenzial aufbaut, das an die Stelle von didaktisch aufbereiteten Meinungsbekundungen und vorgefassten Urteilen die Produktion von Ambivalenz und Strategien der Verunsicherung setzt.« (Ebd.)

Was den Rezipienten an möglichen, aber nicht notwendigen tools bleibt, um Sinn zu konstruieren, sind Recherchen oder aber das Erkennen intertextueller Bezüge. Der Vollständigkeit halber sei erwähnt, dass dabei nicht auf nur eine Deutung hin gelesen werden sollte. Gerade die Deutungsoffenheit ist maßgeblich, damit »aktiv gelesen « werden kann und Bedeutungen gesellschaftlich ausgehandelt werden können (Goer 2003: 172). Diese entsteht nicht nur durch die bereits zu Beginn erwähnten popästhetischen Verfahren, sondern ebenso durch Wortspiele, die verstanden werden können als

»Weigerung[en], sich einer sprachlichen Ordnung zu unterwerfen, eine momentane Taktik, durch die das sprachliche System geplündert und auf respektlose, listige Weise ausgenutzt wird. [...] Geschriebene Sprache ist linear, ihre Beziehungen sind logisch und verbunden durch die Gesetze von Ursache und Wirkung. Wortspiele sind assoziativ, sie entkommen diesen Gesetzen, denn assoziative Beziehungen sind wesentlich freier als logische. [...] Vielmehr beziehen sie parallele Prozesse ein, die Fähigkeit, simultan verschiedene Informationsflüsse zu verarbeiten. [...] Sie erfordern produktives Lesen [...].«(Fiske 2008: 46) 
Das Wortspiel nutzt Neumeister somit als eine weitere Methode, um die Linearität des Textes zu stören (»Darstellung // Gegendarstellung // [...] // Darstellung gegen Darstellung « [AD: 24]). Der Leser muss hierbei >mitspielen $<$ d.h. produktiv lesen, und sich auf die assoziative, teils willkürlich anmutende Reihenfolge der Sätze einlassen. Wie folgendes Zitat demonstriert, lässt sich zwar oftmals kein inhaltlicher Zusammenhang erkennen, doch könnte dies ein Hinweis darauf sein, dass der Erzähler auf diese Weise die von Fiske erwähnte Simultaneität herzustellen versucht, die sich aus der gleichzeitigen Verarbeitung verschiedener Informationsflüsse (Fernsehen, Printmedien, Internet) ergibt (vgl. Fiske 2008: 46).

»automatische Kameras

ferngesteuerte Kameras //

mobile Kameras

immobile Kameras

die Würde des Menschen ist unfaßbar //

Abb.: Andrew WK beim Interviewtermin in der Ritze //

(this site is so sick the government wants so ban it)«(AD: 73)

Aufgrund der Aneinanderreihung unterschiedlicher Themen aus verschiedenen Medien - in dieser Passage wären dies das Fernsehen (angedeutet durch die »Kameras «), Printmedien (angedeutet durch das Kürzel »Abb.«) und Internetseiten (angedeutet durch das englische Wort $>$ site $<$ ) - entsteht der Eindruck, dass diese drei Medien gleichzeitig rezipiert werden. Den Lesern wird mit dieser Vorgehensweise ein Höchstmaß an Konzentration abverlangt, die jederzeit in Resignation kippen kann. An anderer Stelle in Angela Davis löscht ihre Website werden Songlyrics in den Text integriert und mit TV-Slogans kompiliert, um den Eindruck der Gleichzeitigkeit entstehen zu lassen:

»we know how to entertain you!

we have ways of making you dance!

we know how to entertain you!

we have ways of making you dance!

we know how to entertain you!

we have ways of making you dance!

we know how to entertain you!

we have ways of making you dance!«(AD: 30) 
Der Liedtext Unite von den Tiger Tunes, einer Band, die 2001 durch ihre im Internet verbreitete Musik bekannt wurde (»we have ways of making you dance!«), wird mit dem Werbeslogan der Mediengruppe Kirch (»we know how to entertain you! ) vermischt und eröffnet infolgedessen eine Reihe von Deutungspotenzialen.

Die zahlreichen Grenzüberschreitungen in Angela Davis lassen sich anhand der vorangegangenen Beispiele besonders anschaulich nachvollziehen. Nicht nur wird die Unterscheidung von realer Wirklichkeit und Fernsehwirklichkeit, von Genrebezeichnungen und von Boulevard- und Qualitätsjournalismus abgeschafft, ebenso wenig werden medienspezifische Eigenschaften berücksichtigt. Internet, Printmedien, Fernsehen, Musik - alle werden im Medium Buch gesammelt und zu einer - der Popliteratur gern attestierten - ,Oberfläche` zusammengefasst. Was jedoch nach Simplifizierung klingt, erwirkt einen gegenteiligen Effekt. Die vereinfachten und logisch angeordneten Medieninhalte werden vermischt und in einen uneindeutigen Text übersetzt, der dementsprechend ein produktives Lesen einfordert. Die zentrale Rolle der Rezipienten liegt somit auf der Hand. Ebenso wie das »Fernsehen als ein ganzheitliches System von Sprache und Kommunikation im Zusammenspiel mit seinem Publikum« (Renger 2009: 228) gesehen werden muss, kann auch Neumeisters Text als ein solches System bezeichnet werden. Im Gegensatz zu Sapir und Whorf entwirft Neumeister keine Hierarchie im Sinne von >geeigneteren< oder > ungeeigneteren< Medien, weder bestimmte Text- noch Bildformen lehnt er ab. Dafür sprechen auch andere Texte des Autors wie bspw. der 1998 erschienene Roman Gut laut sowie der Aufbau seiner Homepage, die ausschließlich aus scheinbar willkürlich verlinkten (italienischen) Pressefotos und Bilduntertiteln besteht. ${ }^{8}$

\section{Im Zweifelsfall immer Musik hören?}

Schlussendlich zeigt Neumeister mit seiner Collage, dass die Bezeichnung derjenigen Reality-Formate, die in den 1990ern boomten, ebenso obsolet ist wie die Differenzierung zwischen »fiction« und »non-fiction« (AD: 45). Mit dem Vorschlag des Erzählers, die Fernsehrealität als »non-fictive fiction« (AD: 20) zu definieren, führt er diese Genreeinteilung ad absurdum. Betont wird folglich zunächst, was jeder reflektierte Rezipient weiß, nämlich dass jede Medien-Realität eine konstruierte ist. Die Aussage, dass das Fernsehen mit Big Brother zum Medium der totalen Enthüllung (vgl. Postman 1996) geworden sei, da der wirkliche

8 Vgl. www.andreas-neumeister.de/pages/presse_terrazzo.html 
Alltag der Container-Bewohner über millionen Bildschirme zu verfolgen war, erfährt somit eine Entdramatisierung. Nichtsdestoweniger hat das polarisierende Format Big Brother die Medienlandschaft nachhaltig geprägt und unter Beweis gestellt, dass Fernsehen einen nennenswerten Einfluss auf kulturelle Prozesse hat. Der Simulationsmaschine Fernsehen wird insofern ein Machtpotenzial zugeschrieben, als sie mittels Bildmontage und eigens für das TV produzierten Sendungen zum Mitveranstalter ihrer eigenen Realität wird. Diese wiederum bedingt das Entstehen einer populären Realität.

Neumeister fokussiert sich - vielleicht sogar aus eben diesem Grunde - umso stärker auf den Appell zur Reflexion: »you decide! « Dieser Ausruf, der als Schlüsselstelle des Textes bezeichnet werden kann, erinnert an John Hartleys »[t]he outcome is up to you « (Hartley 1996: 251), mit dem bekräftigt wird, dass Realität nicht >von Medien gemacht< wird, sondern das Resultat dessen ist, was der Rezipient aufnimmt, reflektiert und weitergibt respektive gesellschaftlich aushandelt.

Vor dieser Folie kann Angela Davis löscht ihre Website trotz kritischer Erzähler-Kommentare weder eine kulturpessimistische Lesart attribuiert werden noch erfährt das Fernsehen als popkulturelles und Populärkultur stiftendes Medium eine Ablehnung. Vielmehr verweist der Erzähler permanent auf dessen Unterhaltungs- und Popularisierungsfunktion, denn diese - und nicht die exakte Darstellung der Wirklichkeit - sind die primären Ziele des TV. Jeder kritischen Infragestellung folgt somit eine affirmative Stellungnahme, der durch das wiederholte »I'm okay« immer wieder Nachdruck verliehen wird. Diese zunächst paradox erscheinenden Positionen unterstreichen auf der einen Seite, dass Popliteratur nicht immer in einem Extrem zu verorten ist, sondern Subversion und Affirmation gleichermaßen bestehen lässt. Auf der anderen Seite können nur auf diese Weise konträre Stellungnahmen wie beispielsweise »Musik ist besser als Fernsehen, im Zweifelsfall immer Musik hören « und »noch nie liefen so viele klasse Filme im Fernsehn [sic!] wie heute [...]« nebeneinander funktionieren, »die Frage ist« lediglich, so konstatiert der Erzähler, »ob man gerade das richtige Programm eingeschaltet hat (Neumeister 2001: 118 u. 178).

\section{Literatur}

Arnold, Heinz L./Schäfer, Jörgen (Hg.) (2003): Pop-Literatur, München: Text+Kritik.

Balke, Friedrich/Schwering, Gregor/Stäheli, Urs (Hg.) (2000): Big Brother. Beobachtungen (= Masse und Medium, Band 1), Bielefeld: transcript. 
Baudrillard, Jean (1994): Die Illusion des Endes oder der Streik der Ereignisse (= Internationaler Merve-Diskurs, Band 184), Berlin: Merve.

Böhn, Andreas/Seidler, Andreas (2014): Mediengeschichte. Eine Einführung, Tübingen: Narr.

Brichta, Mascha (2010): »Zwischen Popularität und Abwertung. Zur Bedeutung der >Bild-Zeitung « im Alltag ihres Publikums«, in: Jutta Röser/Tanja Thomas/Corinna Peil (Hg.), Alltag in den Medien - Medien im Alltag (= Medien - Kultur - Kommunikation), Wiesbaden: VS, S. 202-219.

Engell, Lorenz (2012): Fernsehtheorie zur Einführung (= Schriften des Internationalen Kollegs für Kulturtechnikforschung und Medienphilosophie, Band 10), Hamburg: Junius.

Fiske, John (2005): Reading television, London: Routledge.

Ders. (2008): »Populäre Texte. Sprache und Alltagskultur«, in: Andreas Hepp/ Rainer Winter (Hg.), Kultur - Medien - Macht, 4. Aufl., Wiesbaden: VS, S. 41-60.

Goer, Charis (2003): »Cross the Border - Face the Gap. Ästhetik der Grenzerfahrung bei Thomas Meinecke und Andreas Neumeister«, in: Arnold/ Schäfer, Pop-Literatur, S. 172-182.

Goetz, Rainald (2008): Klage, Frankfurt am Main: Suhrkamp.

Goldbeck, Kerstin/Kassel, Susanne (2000): »Die Containergesellschaft - Big Brother im Spiegel der Feuilletons«, in: Kurt Beck/Frank Weber (Hg.), Big Brother. Inszenierte Banalität zur Prime Time (= Wissenschaftliche Paperbacks, Band 11), Münster: LIT, S. 233-252.

Greif, Stefan (2004): »In Video veritas. Rainald Goetz' videographische Fernsehdokumentation 1989«, in: Felix Holtschoppen/Frank Linden/Friederike Sinning et al. (Hg.), Clips: eine Collage, Münster: LIT, S. 115-132.

Hartley, John (1996): Popular Reality: Journalism, Modernity, Popular Culture, New York: Arnold.

Hicktethier, Knut/Bleicher, Joan Kirsten (1998): »Die Inszenierung der Information im Fernsehen«, in: Herbert Willems/Martin Jurga (Hg.), Inszenierungsgesellschaft. Ein einführendes Handbuch, Wiesbaden: Westdeutscher Verlag, S. 369-384.

Kloock, Daniela/Spahr, Angela (2012): Medientheorien. Eine Einführung, 4. Aufl., Paderborn: Fink.

Mikos, Lothar (2001): »Fernsehen, Populärkultur und aktive Konsumenten. Die Bedeutung John Fiskes für die Rezeptionstheorie in Deutschland «, in: Rainer Winter (Hg.), Die Fabrikation des Populären. Der John Fiske Reader (= Cultural Studies, Band 1), Bielefeld: transcript, S. 361-371.

Neumeister, Andreas (2001): Gut laut 2.0, Frankfurt am Main: Suhrkamp. 
Ders. (2002): Angela Davis löscht ihre Website, Frankfurt am Main: Suhrkamp. Postman, Neil (1996): Wir amüsieren uns zu Tode. Urteilsbildung im Zeitalter der Unterhaltungsindustrie, Frankfurt am Main: Fischer.

Renger, Rudi (2009): »John Hartley: Populärer Journalismus«, in: Andreas Hepp/Friedrich Krotz/Tanja Thomas (Hg.), Schlüsselwerke der Cultural Studies (= Medien - Kultur - Kommunikation), Wiesbaden: VS, S. 224-234.

Rüdenauer, Ulrich (2001): »Andreas Neumeister im Gespräch: Ist Pop mittlerweile zu brav geworden, Herr Neumeister?«, in: Tagesspiegel vom 10.07.2001. Online unter: http://www.tagesspiegel.de/kultur/andreasneumeis ter-im-gespraech-ist-pop-mittlerweile-zu-brav-geworden-herrneumeister/240 514.html

Schumacher, Eckhard (2003): »>Das Populäre. Was heißt denn das?< Rainald Goetz' >Abfall für alle««, in: Arnold/Schäfer, Pop-Literatur, S. 158-171.

Schwering, Gregor/Stäheli, Urs (2000): »Masse und Medium: Der Container und seine Umwelt«, in: Balke/Schwering/Stäheli, Big Brother, S. 7-16.

Stäheli, Urs (2000): »Das Experiment >Authentizität< - zur Interdiskursivität von Versuchsanordnungen «, in: Balke/Schwering/Ders., Big Brother, S. 55-78.

Warhol, Andy/Hackett, Pat (2013): »POPism. Meine 60er Jahre«, in: Charis Goer/Stefan Greif/Christoph Jacke (Hg.), Texte zur Poptheorie, Stuttgart: Reclam, S. 142-155.

Weingart, Brigitte (2005): »In/Out. Bild-Text-Strategien in Pop-Texten der sechziger Jahre«, in: Wilhelm Voßkamp (Hg.), Sichtbares und Sagbares. Text-Bild-Verhältnisse (= Mediologie, Band 14), Köln: Dumont, S. 216-254.

Wills, Wolfram (2001): »Substantivische Wortbildungen in der deutschen Gegenwartssprache«, in: Gerhard Stickel (Hg.), Neues und Fremdes im deutschen Wortschatz. Aktueller lexikalischer Wandel, Berlin: De Gruyter, S. 172-182.

\section{Medien}

Andreas Neumeisters Homepage: www.andreas-neumeister.de/pages/presse_ terrazzo.html

\section{Abbildungen}

Abbildung 1: Kameralinse mit Big Brother-Logo, Quelle: www.youtube.com/ watch? $\mathrm{v}=\mathrm{WTmbCKsVi90}$ 